\title{
Preoperative predictive model of surgical planning in endometrial carcinoma using MRI and tumor grade in endometrial biopsy
}

\author{
Mikel Gorostidi ${ }^{1,2 *}$, Ruben Ruiz ${ }^{1}$, Ibon Jaunarena ${ }^{1,2}$, Paloma Cobas ${ }^{1}$ Arantxa Lekuona ${ }^{1,2}$ and Irene Diez ${ }^{1,2}$ \\ ${ }^{1}$ Departamento de Obstetricia y Ginecología, Hospital Universitario Donostia, P. Beguiristain s/n, 20014 San Sebastián Spain. \\ ${ }^{2}$ BIODONOSTIA Health Research Institute, San Sebastián, Spain.
}

*Corresponding Author: Mikel Gorostidi Departamento de Obstetricia y Ginecología, Hospital Universitario Donostia, P. Beguiristain s/n, 20014 San Sebastián Spain.

Received date: March 09, 2021; Accepted date: March 13, 2020; Published date: March 20,2021

Citation: Gorostidi M., Ruiz R., Jaunarena I., Cobas P., Diez I. and Lekuona A. (2021) Preoperative predictive model of surgical planning in endometrial carcinoma using MRI and tumor grade in endometrial biopsy J. Obstetrics Gynecology and Reproductive Sciences 5(2); DOI: $10.31579 / 2578-8965 / 065$

Copyright: ( ) 2021, Mikel Gorostidi, This is an open access article distributed under the Creative Commons Attribution License which permits unrestricted use, distribution and reproduction in any medium provided the original work is properly cited.

\begin{abstract}
Introduction: Endometrial cancer (EC) is the most common gynecological cancer in developed countries. Histological grade $(\mathrm{G})$ and myometrial invasion (MI) are important risk factors, and together with the histological type and other postoperative data establish the risk of lymph node involvement and guide the adjuvant treatments. The objective of this study was to assess the validity of a preoperative stratification model that combines preoperative histological grade and MI as identified by magnetic resonance imaging (MRI) to select candidates for lymph node staging and optimize surgical planning for our patients.
\end{abstract}

Material and methods: It's an observational retrospective cohort study including 294 patients diagnosed with EC at Donostia University Hospital from January 2012 to December 2017. Preoperative endometrial biopsy, including histological type and grade, preoperative MRI was compared with the definitive histological diagnosis. Sensitivity (Sn), specificity (Sp), positive predictive value (PPV) and negative predictive value (NPV) of the MRI-based diagnosis were calculated.

Results: After inclusion and exclusion criteria 242 cases of type I or II EC were analyzed. Our model was found to have a Se of 91.4\% (95\% CI 83.2-95.8) and a Sp of 90.7\% (95\% CI 85.2-94.3). Percentage of down staging was 6.2\% (15 unnecessary lymphadenectomies) and the upstaging rate was $2.9 \%$. The NPV of the model was very high $(95.4 \%, 95 \%$ CI 90.9-97.8). The diagnostic odds ratio for our model was 147.95 (95\% CI 52.9-410.5), with a diagnostic accuracy of $91.7 \%$ (95\% 87.6-94.6).

Conclusions: A preoperative strategy that includes the determination of the tumor grade based on an endometrial biopsy and an assessment of MI by MRI is of great help in pre-surgical planning for endometrial cancer surgery, allowing an extra peritoneal approach and optimizing the use of physical and human resources. MRI presents excellent discriminatory power in the detection of MI in EC, with no significant variation by pathological subtype.

Keywords: endometrial carcinoma; MRI; tumor grade; endometrial biopsy

\section{Introduction}

Endometrial cancer is the most common gynecological cancer in developed countries [1] with a standardized incidence of 13.6 per 100,000 women and more than 100,000 new cases diagnosed per year in Europe. In Spain, it is the most common female genital tract malignant tumor and ranks second in mortality, after ovarian cancer. Its incidence is 5.9 per 100,000 women, with a mortality rate of 3.1 per 100,000 and a 5 -year prevalence of 7.6 per 100,000. Most cases are diagnosed in early stages $(80 \%$ in stage I), with a 5-year overall survival of $95 \%$. Nonetheless, survival is significantly shorter in cases of regional dissemination or distant disease, resulting in even lower survival rates than in ovarian cancer at equally advanced stages (III and IV).

Histological grade (G) and myometrial invasion (MI) influence the risk of lymph node involvement, this increasing from $1 \%$ in cases of G1-2 tumors with invasion of less than $50 \%$, to more than $10 \%$ in cases of higher grade tumors or a greater extent of invasion. The grade has been shown to determine the probability of survival in women with stage I or II endometrioid endometrial cancer; with 5-year survival rates of 93\%, $85 \%$ and $69 \%$ in G1, G2 and G3 respectively [3] Notably, within G1, MI is associated with lower survival: $87 \%$ vs. $99 \%$ when there is no MI.

The recommended imaging tests for presurgical risk stratification 
through assessment of uterine risk factors (size, myometrial invasion, cervical stromal involvement and involvement of the lower uterine third) are magnetic resonance imaging (MRI) and transvaginal/transrectal ultrasound, depending on their availability [3]. There is no evidence of the superiority of any specific approach, they are operator dependent, and they show an agreement of around $85 \%$ with the definitive histological diagnosis, and hence, where both are available, the decision of which to use is left to each center based on its results. The diagnostic accuracy of MRI, assessed against a definitive histological examination, has been found to be between $71 \%$ and $97 \%$ [4].

Our objective was to assess the validity of a preoperative stratification model that combines preoperative histological grade and MI as identified by MRI to select candidates for lymph node staging and optimize surgical planning for our patients.

\section{Material and methods}

An observational retrospective cohort study was conducted including 294 patients diagnosed with endometrial carcinoma at Donostia University Hospital from January 2012 to December 2017. All patients were histologically diagnosed with endometrial carcinoma by preoperative endometrial biopsy, including histological type and grade. All patients underwent preoperative magnetic resonance imaging (MRI). Data were obtained from electronic health records.

Cases of early stage endometrial carcinoma (I and II) with indications for surgery were selected for inclusion. Cases were excluded if the MRI yielded an inconclusive result concerning MI or we failed to obtain any of the relevant data from the health records. The diagnosis of concomitant uterine pathology and intrauterine location of the tumor were not taken into account. Work-up and different diagnosis tests performed are shown in figure 1.

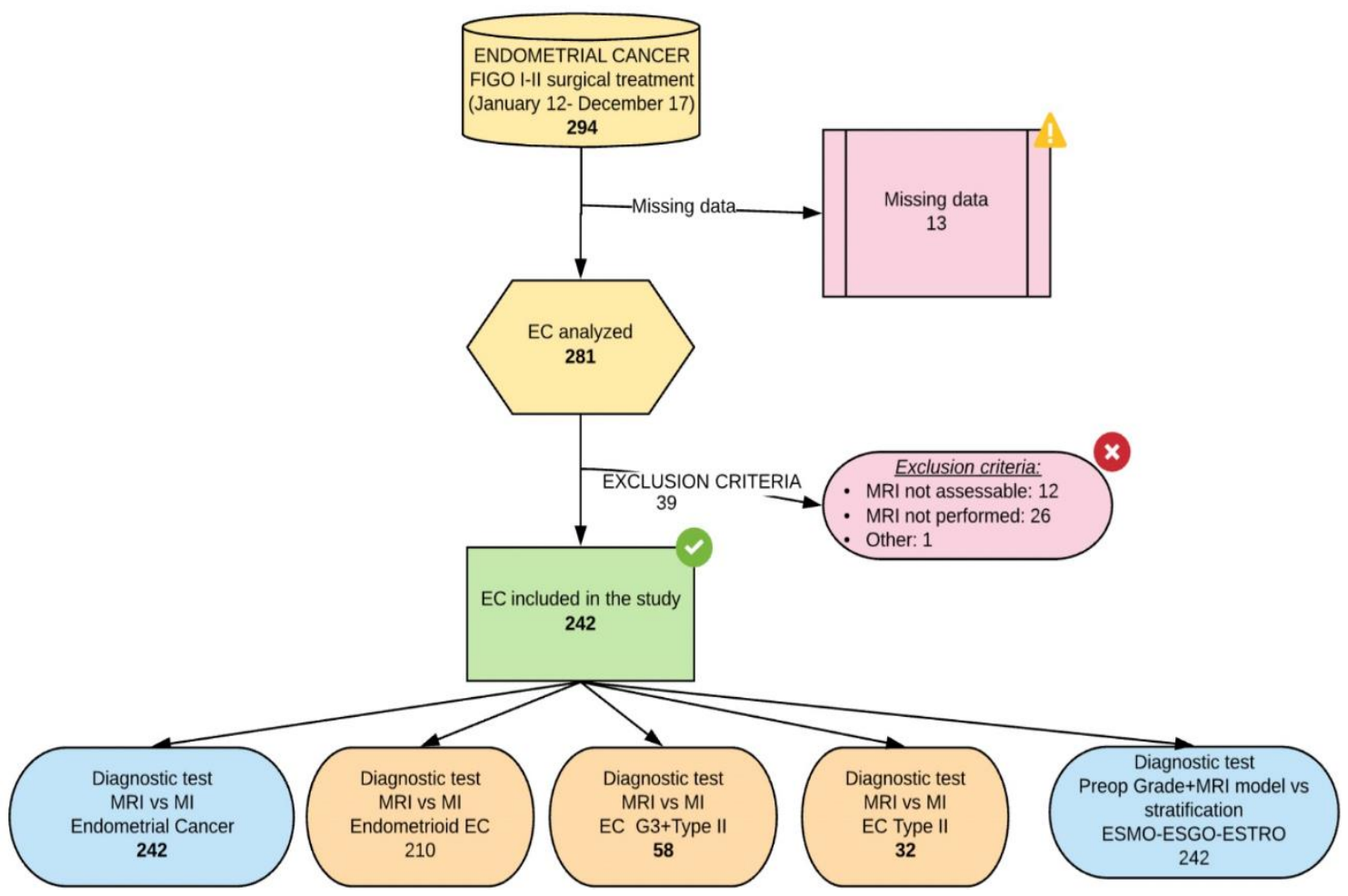

Figure 1. Work-up flow Chart

Preoperative results were compared with the definitive histological diagnosis from analysis of the hysterectomy specimen. Stratified analysis was performed for cases with the endometrioid subtype and for type II CE. MRI and post-surgical staging were compared in terms of the extent of MI (greater than or equal to or less than 50\%). Sensitivity (Sn), specificity $(\mathrm{Sp})$, positive predictive value (PPV) and negative predictive value (NPV) of the MRI-based diagnosis were calculated.

Further, a model was developed comparing preoperative stratification based on preoperative grade and MRI findings with final postoperative stratification (in accordance with ESMO-ESGO-ESTRO (European Society for Medical Oncology - European Society of Gynecological Oncology - European Society for Radiotherapy \& Oncology) recommendations) based on tumor grade, MI and lymphovascular space involvement (LVSI), considering tumor grade 3 and LVSI to be true positives. The diagnostic performance of this model was assessed. Approval by the hospital ethics committee (IRB) was obtained.

All variables were entered into an Excel form designed for this purpose and analyzed using Stata 15.0 statistical software (STATA Corp LCC, TX, USA). Quantitative variables are presented as median and standard deviation (SD) and categorical variables as proportions. As well as Se, $\mathrm{Sp}, \mathrm{PPV}, \mathrm{NPV}$, positive and negative likelihood ratios (LH+ and LH-, respectively) with $95 \%$ Wilson confidence intervals (CIs) were calculated to assess the performance of the model. The accuracy of the model was also estimated.

\section{Results}

There were a total of 294 cases of endometrial cancer during the study 
period. Of these, 13 cases were excluded due to missing information, while in another 39 patients, MRI had not been performed or the findings were inconclusive. Finally, a total of 242 cases of type I or II endometrial carcinoma were analyzed. The mean age of these patients was 64.7 years (SD 10.76). They had a mean body mass index of $29.5 \mathrm{~kg} / \mathrm{m}^{2}$ (SD 6.94) and a mean tumor size of $29.5 \mathrm{~mm}$ (SD 18.4).
An Se of $77.8 \%$ (67.6-85.5), Sp of $86.3 \%$ (80.2-90.8), PPV of $74.1 \%$ (63.9-82.2) and NPV of 88.5\% (82.6-92.6) were obtained for all types of endometrial cancer and MRI assessment as a diagnostic test for MI, with a prevalence of $33.5 \%$ of endometrial cancer cases with $\mathrm{MI} \geq 50 \%$, an $\mathrm{LH}+$ of 5.69 (3.78-8.54) and an LH- of $0.26(0.17-0.39)$. Table 1

\begin{tabular}{|c|c|c|c|c|c|}
\hline & $\begin{array}{l}\text { MRI vs } \\
\text { Histological MI } \\
\text { All types of EC }\end{array}$ & $\begin{array}{l}\text { MRI vs Histological } \\
\text { MI } \\
\text { Endometrioid EC }\end{array}$ & $\begin{array}{l}\text { MRI vs Histological } \\
\text { MM } \\
\text { G3+Type II EC }\end{array}$ & $\begin{array}{l}\text { MRI vs Histological } \\
\text { MI } \\
\text { Type II EC }\end{array}$ & $\begin{array}{l}\text { Model vs ESMO-ESGO- } \\
\text { ESTRO stratification }\end{array}$ \\
\hline & $\begin{array}{l}\text { Estimated } \% \\
(95 \% \text { CI })\end{array}$ & $\begin{array}{l}\text { Estimated \% }(95 \% \\
\text { CI) }\end{array}$ & $\begin{array}{l}\text { Estimated \% } \\
(95 \% \mathrm{CI})\end{array}$ & $\begin{array}{l}\text { Estimated \% } \\
(95 \% \mathrm{CI})\end{array}$ & $\begin{array}{l}\text { Estimated \% } \\
(95 \% \mathrm{CI})\end{array}$ \\
\hline Sensitivity & $77.8(67.6-85.5)$ & $79.7(66.8-87.5)$ & $72(52.4-85.7)$ & $79.3(48-89.1)$ & $93.8(86.4-97.3)$ \\
\hline Specificity & $86.3(80.2-90.8)$ & $85.1(78.3-90)$ & $84.8(69.1-93.3)$ & $91.3(73.2-97.6)$ & $90.7(85.2-94.3)$ \\
\hline False positive & $13.7(9.2-19.8)$ & $14.9(9.95-21.7)$ & $15.2(6.65-30.9)$ & $8.7(2.42-26.8)$ & $9.32(5.73-14.8)$ \\
\hline False negative & $22.2(14.5-32.4)$ & $20.3(12.5-31.2)$ & $28(14.3-47.6)$ & $26.7(10.9-52)$ & $6.17(2.67-13.6)$ \\
\hline $\begin{array}{l}\text { Positive } \\
\text { likelihood ratio }\end{array}$ & $5.69(3.79-8.53)$ & $5.35(3.54-8.08)$ & $4.75(2.04-11.04)$ & $8.43(2.17-32.82)$ & $10.07(6.2-16.36)$ \\
\hline $\begin{array}{l}\text { Negative } \\
\text { likelihood ratio }\end{array}$ & $0.26(0.17-0.39)$ & $0.24(0.15-0-38)$ & $0.33(0.17-0.62)$ & $0.29(0.13-0.68)$ & $0.07(0.03-0.16)$ \\
\hline Odds ratio & $22.1(11.1-43.9)$ & $22.5(10.7-47.2)$ & $14.4(4.05-51.07)$ & $28.8(4.9-164)$ & $147.95(52.9-410.5)$ \\
\hline $\begin{array}{l}\text { Sample } \\
\text { prevalence }\end{array}$ & $33.5(27.8-39.6)$ & $32.9(26.9-39.5)$ & $43.1(31.2-55-9)$ & $39.5(25.6-55.3)$ & $33.5(27.8-39.6)$ \\
\hline $\begin{array}{l}\text { Positive } \\
\text { predictive value }\end{array}$ & $74.1(63.9-82.2)$ & $72.4(61.4-81.2)$ & $78.3(58.1-90.3)$ & $84.6(57.8-95.7)$ & $83.5(74.6-89.7)$ \\
\hline $\begin{array}{l}\text { Negative } \\
\text { predictive value }\end{array}$ & $88.5(82.6-92.6)$ & $89.6(83.2-93.7)$ & $80(64.1-90)$ & $84(65.3-93.6)$ & $96.7(92.5-98.6)$ \\
\hline $\begin{array}{l}\text { Diagnostic } \\
\text { accuracy }\end{array}$ & $83.5(78.3-87.6)$ & $83.3(77.7-87.8)$ & $79.3(67.2-87.7)$ & $84.2(69.6-92.6)$ & $91.7(87.6-94.6)$ \\
\hline
\end{tabular}

MRI: magnetic resonance imaging, EC: endometrial cancer, MI: myometrial invasion

Table 1. Performance of the diagnostic test models in the different situations considered.

There were 22 false positive cases, among which there were 5 cases of G3 and 4 cases of LVSI (+), 2 cases both being G3 and having LVSI (+), and hence, only 15 cases were actually down staged after the definitive histological diagnosis. Regarding false negatives, these included seven cases of G3 tumors and eight cases of LVSI (+), with three of the latter being G3. Further, in one case, stromal invasion was diagnosed by MRI and this was histologically confirmed. That is, there were 13 false negatives from MRI in this series and these corresponded to G3 and/or LVSI (+) tumors or stromal involvement.

In the case of endometrioid histology, there were 21 false positives and 14 false negatives in a total of 210 patients. The $\mathrm{Sn}$ was $79.7 \%$, Sp $85.1 \%$, PPV $72.4 \%$ and NPV $89.6 \%$. The Sn and Sp of the test were found respectively to be $72 \%$ and $84.8 \%$ in the case of type II tumors (including G3 endometrioid, clear cell and serous carcinoma) and 73.3\% and 91.3\% in the case of undifferentiated carcinoma, carcinosarcoma, and mixed, serous and clear cell carcinoma.

When analyzing the performance of our model that includes grade and MRI as a diagnostic test, comparing with the ESMO-ESGO-ESTRO postoperative stratification, the $\mathrm{Sn}$ increased to $93.8 \%$ (95\% CI 86.497.3\%) and the Sp to $90.7 \%$ (95\% CI 85.2-94.3\%). The diagnostic odds ratio for our model was 147.95 (95\% CI 52.9-410.5), with a diagnostic accuracy of $91.7 \%$ (95\% 87.6-94.6).

\section{Discussion}

This study supports the idea of preoperatively planning the type of surgery to be performed, as it seems possible to estimate the definitive result of the histopathological examination with high reliability. The decision to schedule a pelvic and/or aortocaval lymphadenectomy from the outset has numerous advantages and implications. Not only are we able to plan the surgical time in the operating room and optimize its use, we are also able to avoid the use of other resources, for example, omitting intraoperative frozen section analysis, with the associated problems and time delays, and we are able to discuss with patients in advance the most appropriate procedure in their case.

What is more, the main benefit, in our opinion, is the possibility of using an extra peritoneal approach [5], that it would be impossible if we had to base our decision on intraoperative histopathological findings. This is especially important considering the characteristics of patients that we often encounter with this condition, a large proportion of them being obese and/or having comorbidities, which make it difficult and sometimes impossible to reach the root of the mesentery and the Trendelenburg position is required for the transperitoneal approach. It has also been reported that in obese patients fewer nodes are obtained with the transperitoneal route [6,7] and that the number of lymph node metastases found increases with the number of nodes obtained [7], 
although in the only randomized study comparing the two approaches, no significant differences were found in the perioperative results [8].

Our model is based on two key variables, namely, MRI findings and histological tumor grade. The application of this model requires the preoperative histological diagnosis to include tumor grade, which may sometimes be difficult to obtain preoperatively, but which can be obtained with the involvement of a highly motivated multidisciplinary team. In our case, we repeat the endometrial sample in cases for which we do not yet have the tumor grade, either because the biopsy was taken at another hospital or a shortage of sample material, if necessary, by hysteroscopy to improve the quality of the sample taken. It is also vital to have a team of radiologists involved and trained in the assessment of MI, as well as internal monitoring of their results, these undoubtedly resulting in improvements in discriminatory capacity.

If we already have a diagnosis of high-tumor grade, we omit the MRI, since it does not provide any additional useful information and to assess the MI, we perform axial computed tomography (CT) for extended diagnosis. Our objective in such cases is to detect peritoneal disease and anticipate the need for a laparotomy and cytoreduction, which may be more appropriate in stages III and IV than laparoscopic surgery.

Contrast MRI has proven to be a better imaging test to detect MI, cervical stromal involvement and lymph node involvement than MRI without contrast, ultrasound or CT $[9,10]$. Fusion of T2-weighted magnetic resonance imaging (T2WI) and diffusion-weighted magnetic resonance imaging (DWI) may contribute to the improvement of anatomical localization and assessment of myometrial invasion [11, 12]. In our series, MRI showed a high NPV of $88.5 \%$, although its PPV of $74.1 \%$ was lower than expected, which would explain the cases of over diagnosis with this test; and this was substantially improved by applying our model that also includes the preoperative histological grade, the PPV increasing to $83.1 \%$.

LVSI has proven to be an independent risk factor for local and distant recurrence, as well as an adverse prognostic factor for recurrence, in terms of disease-free survival, overall survival and disease-specific survival, with hazard ratios (HRs) of 2.8, 2.8 and 7 respectively. It has even been suggested that LVSI should be included in the FIGO classification, since it has been seen that patient survival is even better predicted in isolation with this factor, with rates of $81 \%$ in positive cases and $97 \%$ in negative cases, than considering whether there is MI of greater or less than $50 \%$, with rates of $87 \%$ and $96 \%$ respectively [11]. LVSI is strongly associated with lymph node metastasis [12]; nevertheless, not all groups perform a lymphadenectomy in patients with this type of involvement [13], although it is associated with a change in risk when performing adjuvant treatments [14]. For this reason, our group performs lymph node staging in these patients and we do not consider that patients with final LVSI (+), in spite of low grade and MI less than $50 \%$, are overtreated in our series. On the other hand, LVSI is always assessed postoperatively, meaning that it cannot be included in preoperative stratification models.

Our model was found to have a Se of $91.4 \%$ (95\% CI 83.2-95.8) and an Sp of $90.7 \%$ (95\% CI 85.2-94.3), superior to those with the use of MRI alone and thus improving its diagnostic accuracy. Considering our model, we have performed 15 unnecessary lymphadenectomies in a total of 242 patients, which corresponds to $6.2 \%$ of the sample and represents the rate of overdiagnosis and downstaging in the definitive histological stratification. On the other hand, only seven patients were underdiagnosed, corresponding to an upstaging rate of $2.9 \%$ in our series, and they underwent a lymphadenectomy in a second surgical session. It should be taken into account that one of these cases was a woman who had extramural MRI findings, where the images had been interpreted by radiologists in a small hospital who -unlike the radiologists involved in our team- were not specialized in gynecological tumors.

In our case, today, such patients would no longer undergo reoperation if they had had a sentinel lymph node biopsy and sentinel node involvement had been detected, using our combined cervical and fundal injection technique and pelvic and aortic sentinel node identification [15]. We do not reoperate on such patients in accordance with an individualized decision taken in a tumor committee motivated by our excellent results and the complexity of dissecting a pelvic and especially aortic area for a second time, with adhesions and obliteration being very common and the very high risk of vascular injury, as well as the delay in the start of adjuvant therapy that would imply.

The NPV of the model was very high (95.4\%, 95\% CI 90.9-97.8), indicating that our surgical planning is excellent. Notably, it is very rare that the surgical time needed is longer than that planned, since the results of intraoperative frozen section analysis, which we always perform in patients with G1-2 endometrioid endometrial cancer and MI less than $50 \%$ as assessed by MRI, almost always agree with the previous diagnosis.

Nonetheless, this study has some limitations. No data were recorded regarding the presence of factors that could modify the diagnostic accuracy of MRI such as myometrial lesions, for example, myomas; polypoid tumors; or corneal involvement. Likewise, tumor grade data may vary in provisional and definitive histological diagnoses due to intratumoral heterogeneity.

\section{Conclusions}

A preoperative strategy that includes the determination of the tumor grade based on an endometrial biopsy and an assessment of MI by MRI is of great help in pre-surgical planning for endometrial cancer surgery, allowing an extra peritoneal approach and optimizing the use of physical and human resources. MRI presents excellent discriminatory power in the detection of MI in endometrial cancer, with no significant variation by pathological subtype.

\section{Disclosure}

The authors declare no conflict of interest. None of the authors has any relationship or funding received for this work from.

\section{Acknowledgement}

To Gynecological oncology unit and to all women that have taken part in this research.

\section{References}

1. Siegel RL, Miller KD, Jemal A._(2020) Cancer statistics. CA Cancer J Clin. 70(1):7-30.

2. Zaino RJ, Kurman RJ, Diana KL, Morrow CP._(1995) The utility of the revised International Federation of Gynecology and Obstetrics histologic grading of endometrial adenocarcinoma using a defined nuclear grading system. A Gynecologic Oncology Group study. Cancer. 75(1):81-6.

3. Alcázar JL, Orozco R, Martinez-Astorquiza Corral T, Juez L, Utrilla-Layna J, Mínguez JA, et al. (2015) Transvaginal ultrasound for preoperative assessment of myometrial invasion in 
patients with endometrial cancer: a systematic review and metaanalysis. Ultrasound Obstet Gynecol 46(4):405-13.

4. Frei KA, Kinkel K. (2019) Staging endometrial cancer: role of magnetic resonance imaging. J Magn Reson Imaging. 13(6):8505.

5. Gorostidi M, Villalain C, Ruiz R, Jaunarena I, Lekuona A. (2018) Technique for precaval and laterocaval nodes excision at extraperitoneal paraaortic lymphadenectomy. Gynecol Oncol. 148(1):233-4.

6. Pakish J, Soliman PT, Frumovitz M, Westin SN, Schmeler KM, Reis R dos, et al. (2014) A comparison of extraperitoneal versus transperitoneal laparoscopic or robotic para-aortic lymphadenectomy for staging of endometrial carcinoma. Gynecol Oncol. 132(2):366-71.

7. O'Hanlan KA, Sten MS, O'Holleran MS, Ford NN, Struck DM, McCutcheon SP. (2015) Infrarenal lymphadenectomy for gynecological malignancies: Two laparoscopic approaches. Gynecol Oncol. 139(2):330-7.

8. Díaz-Feijoo B, Correa-Paris A, Pérez-Benavente A, FrancoCamps S, Sánchez-Iglesias JL, Cabrera S, et al. (2016) Prospective Randomized Trial Comparing Transperitoneal Versus Extraperitoneal Laparoscopic Aortic Lymphadenectomy for Surgical Staging of Endometrial and Ovarian Cancer: The STELLA Trial. Ann Surg Oncol. 23(9):2966-74.

9. Kinkel K, Kaji Y, Yu KK, Segal MR, Lu Y, Powell CB, et al. (2019) Radiologic staging in patients with endometrial cancer: a meta-analysis. Radiology. 212(3):711-8.

10. Yin X-H, Jia H-Y, Shi M, Wu H, Li Y-M. (2015) Magnetic resonance imaging for detection of depth of myometrial invasion and cervical invasion in patients with endometrial carcinoma. Int J Clin Exp Med. 8(10):19501-5.
11. Guo Y, Wang P, Wang P, Gao W, Li F, Yang X, et al. (2017) Myometrial invasion and overall staging of endometrial carcinoma: assessment using fusion of T2-weighted magnetic resonance imaging and diffusion-weighted magnetic resonance imaging. Onco Targets Ther. 10:5937-43.

12. Lin G, Ng K-K, Chang C-J, Wang J-J, Ho K-C, Yen T-C, et al. (2009) Myometrial invasion in endometrial cancer: diagnostic accuracy of diffusion-weighted 3.0-T MR imaging--initial experience. Radiology. 250(3):784-92.

13. Aristizabal P, Graesslin O, Barranger E, Clavel-Chapelon F, Haddad B, Luton D, et al. (2014) A suggested modification to FIGO stage I endometrial cancer. Gynecol Oncol. 133(2):192-6.

14. Solmaz U, Mat E, Dereli M, Turan V, Gungorduk K, Hasdemir P, et al. (2019) Lymphovascular space invasion and cervical stromal invasion are independent risk factors for nodal metastasis in endometrioid endometrial cancer. Aust New Zeal J Obstet Gynaecol. 55(1):81-6.

15. Gorostidi Pulgar M, Diaz de la Noval B, Gil-Ibañez B, Lago Leal V, Jaunarena Marin I, Padilla Iserte P. (2017) Lymphovascular Space Invasion for Endometrial Cancer: Undertreatment and Overtreatment Risks: A Survey of the Spanish Gynecologic Oncology Group. Int J Gynecol Cancer. 27(6):1191-9.

16. Colombo N, Creutzberg C, Amant F, Bosse T, González-Martín A, Ledermann J, et al. (2015) ESMO-ESGO-ESTRO consensus conference on endometrial cancer: Diagnosis, treatment and follow-up. Radiother Oncol. 117(3).

17. Ruiz R, Gorostidi M, Jaunarena I, Goiri C, Aguerre J, Lekuona A. (2018) Sentinel node biopsy in endometrial cancer with dual cervical and fundal indocyanine green injection. Int $\mathrm{J}$ Gynecol Cancer.

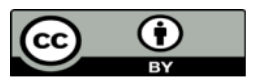

This work is licensed under Creative Commons Attribution 4.0 License

\section{To Submit Your Article Click Here: Submit Manuscript}

DOI: $10.31579 / 2578-8965 / 065$

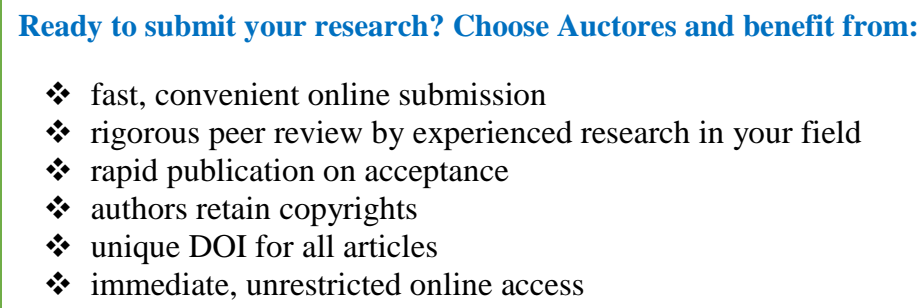

At Auctores, research is always in progress.

Learn more www.auctoresonline.org/journals/obstetrics-gynecology-andreproductive-sciences 